Boise State University

ScholarWorks

3-14-2008

\title{
Cantilever Based Optical Interfacial Force Microscope
}

Jeremy R. Bonander

Boise State University

Byung I. Kim

Boise State University 


\title{
Cantilever based optical interfacial force microscope
}

\author{
Jeremy R. Bonander and Byung I. Kim ${ }^{\text {a) }}$ \\ Department of Physics, Boise State University, Boise, Idaho 83725, USA
}

(Received 15 February 2008; accepted 25 February 2008; published online 14 March 2008)

\begin{abstract}
We developed a cantilever based optical interfacial force microscopy (COIFM) that employs a microactuated silicon cantilever and optical detection method to establish the measurement of the single molecular interactions using the force feedback technique. Through the direct measurement of the COIFM force-distance curves, we have demonstrated that the COIFM is capable of unveiling structural and mechanical information on interfacial water at the single molecular level over all distances between two hydrophilic surfaces. (C) 2008 American Institute of Physics.
\end{abstract}

[DOI: $10.1063 / 1.2898524]$

Atomic force microscopy (AFM) is one of the most important tools that leads current nanoscience and nanotechnology in many diverse areas including physics, chemistry, material engineering, and nanobiology. The current AFM technique has been routinely applied to forced unbinding processes of biomolecular complexes such as antibodyantigen binding, ligand-receptor pairs, protein unfolding, DNA unbinding, and RNA unfolding studies. ${ }^{1-3}$ However, its usage has been limited to passive applications (e.g., pull-off force measurement in the force-distance curve) due to the persistent mechanical instability of cantilever assembly near a sample surface. The mechanical instability causes missing data point near the sample surface due to the rapid snap-in process ${ }^{4}$ in which the force derivative (i.e., $d F_{a} / d z$ ) with respect to the tip position $(z)$ exceeds the stiffness of the cantilever (spring constant $k$ ). ${ }^{5-8}$

Force feedback techniques are capable of preventing the mechanical instability associated with the snap-to-contact process during measurement of an AFM force-displacement curve. The force feedback techniques have offered broad control over the cantilever behavior and have greatly expanded the applicability of the cantilever to the various problems at interfaces. Interfacial force microscopy (IFM) was developed fifteen years ago to avoid these mechanical instability problems ${ }^{9-11}$ and has contributed to molecular scale understanding of various surface phenomena. IFM has been applied to diverse interfacial researches including nanotribology, ${ }^{12-14}$ interfacial adhesion, ${ }^{15,16}$ probing of interfacial water structures, ${ }^{17,18}$ and measurements of chemical interactions. ${ }^{19,20}$ However, the technique has not been widely used due to low sensitivity and technical complexity of the electrical-sensing method. The current IFM system uses a relatively bigger tip with the typical diameter around $1 \mu \mathrm{m}$ for measurement of molecular interactions due to the existing low sensitivity issue in electric force detection method of the current "teeter-totter" type of IFM force sensor." The larger tip and the complexity of the electrical detection measurements have limited the use of the IFM as a popular tool to address the issues, especially at the single molecular level. Magnetic force feedback microscopy was developed by attaching a magnet to the end of a cantilever a decade ago. ${ }^{21-23}$ However, the magnetic force feedback requires a tedious

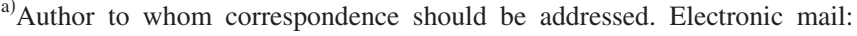
byungkim@boisestate.edu. process of attaching magnets to the backside of the cantilever using an inverted optical microscope equipped with micromanipulators ${ }^{19,23}$ and has poor performance in the servo system due to eddy currents. ${ }^{21-23}$

Here, we report the integration of the existing two scanning-probe techniques (AFM and IFM) through the development of an instrument called a "cantilever based optical interfacial force microscope" (COIFM). The integrated COIFM employs an optical detection method of AFM and a commercially available microactuated silicon cantilever to self-balance the force sensor, which improves the interfacial force sensitivity by an order of magnitude and the spatial sensitivity to the subnanometer scale, enough to resolve the individual water ordering on a silicon surface.

A schematic diagram of the overall COIFM system with the force feedback control is shown in Fig. 1. In the present design, a commercially available cantilever with built-in $\mathrm{ZnO}$ stack called "dimension microactuated silicon probe" (DMASP) is employed as the COIFM sensor (Veeco Instruments). The DMASP serves two separate functions as an active circuit element, i.e., displacement detection and mechanical bending of the $\mathrm{ZnO}$ stack for the voltage activated force feedback (Fig. 1). A nanometer diameter tip underneath the cantilever allows for measuring the intermolecular inter-

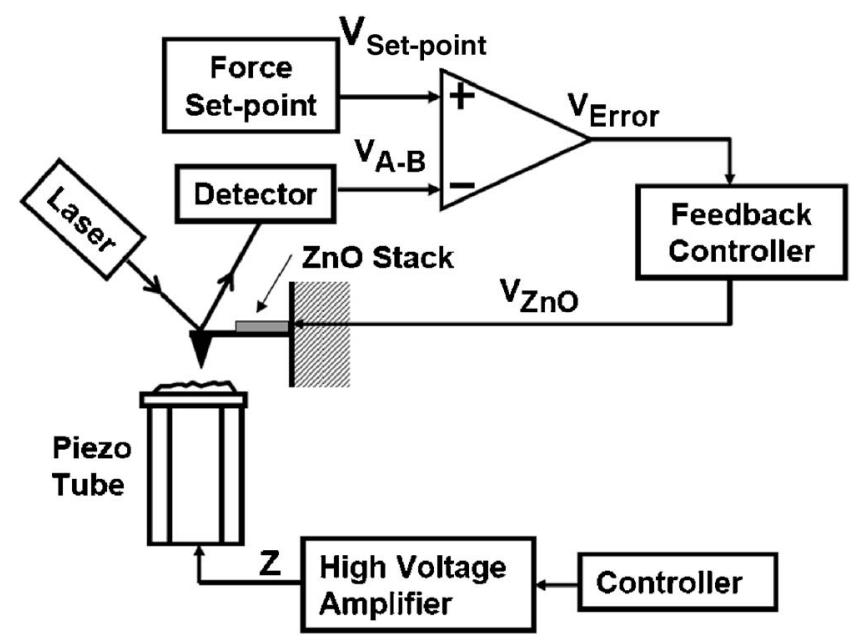

FIG. 1. A schematic diagram of the COIFM with voltage activated force feedback using an optical beam deflection detection method. The system consisted of a LS AutoProbe AFM with a DMASP tip interfaced with a RHK SPM100 controller. 

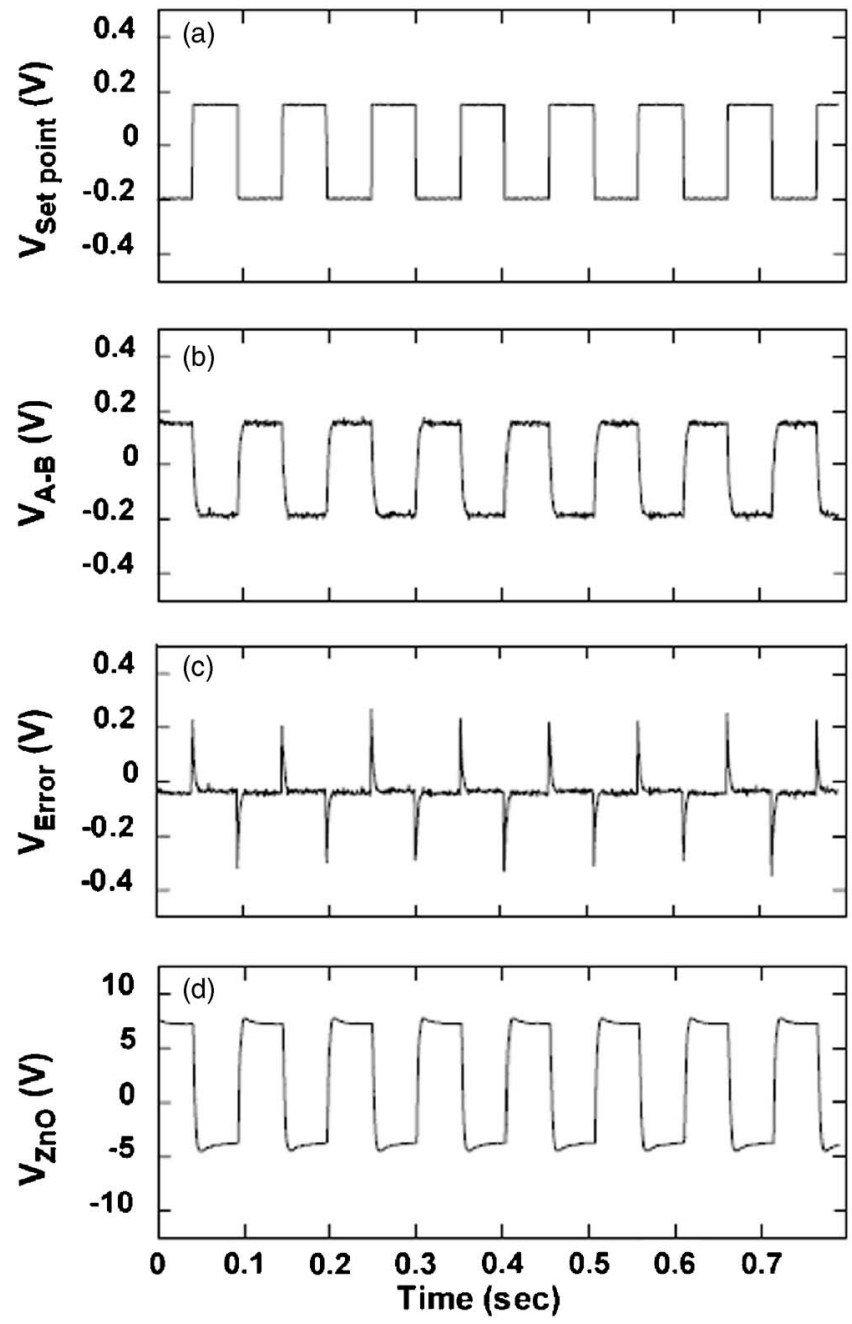

FIG. 2. (a) A square wave ac signal with a frequency of $10 \mathrm{~Hz}$ as a set-point voltage of the feedback loop. (b) The deflection $V_{A-B}$ signal that follows the set-point voltage. (c) The error signal $V_{\text {error }}$ between $V_{\text {set point }}$ and $V_{A-B}$ (d) The signal $V_{\mathrm{ZnO}}$ sent from the controller to the $\mathrm{ZnO}$ stack material.

action at the single molecular level between the tip and a surface. The cantilever has zero compliance during the measurement, thus, preventing the snap-to-contact process associated with typical AFM force-distance measurements. The tip-sample distance in the $z$ direction was controlled by high voltage signal controller sent to the piezotube (RHK Technology, Troy, MI). An optical beam deflection detection scheme in the AFM head of an AutoProbe LS (former Park Scientific Instruments) was used to transmit the interaction force between the tip and the surface into the electrical signal. ${ }^{20}$ The head was interfaced with a RHK SPM 100 controller and all data presented here were recorded through analog-to-digital converter inputs of the RHK 100 and XPM PRO software.

To find the time resolution of the COIFM, a square-wave voltage with amplitude $0.2 \mathrm{~V}$ and frequency $10 \mathrm{~Hz}$ was applied to the set-point voltage $\left(V_{\text {set point }}\right)$ with the force feedback far away from the surface. ${ }^{24}$ Figures 2 (a)-2(d) show that the feedback controller tries its best for the preamp output $\left(V_{A-B}\right)$ to follow this square wave by applying appropriate voltages to the $\mathrm{ZnO}$ stack of the DMASP sensor $\left(V_{\mathrm{ZnO}}\right)$. The square wave causes the cantilever to create a torque on the cantilever so as to achieve a zero error voltage $V_{\text {error }}$ with the feedback on [Fig. 2(c)]. The controller is set up to optimize Downloaded 09 Apr 2009 to 132.178.155.253. Redistribution subje
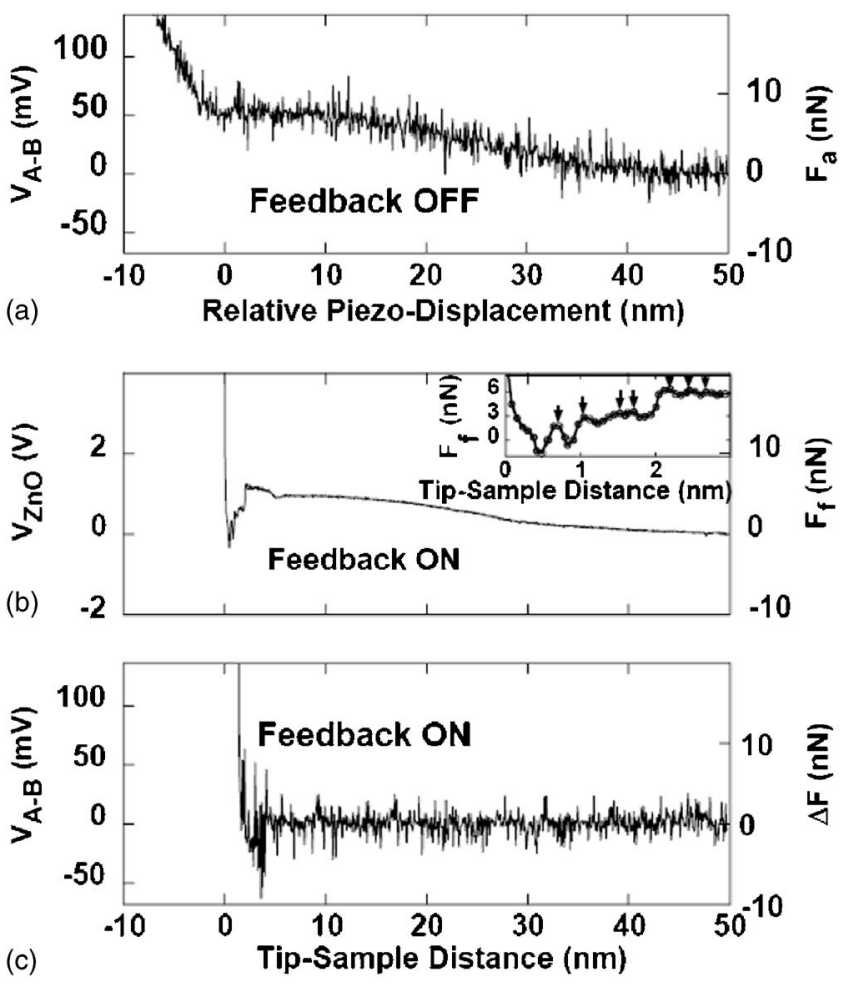

FIG. 3. (a) A force-displacement curve between the tip and the silicon surface obtained without a force activated voltage feedback system. (b) The force applied to the $\mathrm{ZnO}$ stack material graphed as a function of tip and silicon sample distance. (inset) Enlarged force-distance curve between 0 and $3 \mathrm{~nm}$. (c) Force-distance curve between the tip and the silicon surface obtained with a force activated voltage feedback system.

the transient response in order to achieve the necessary time response for a COIFM experiment. The transient feedback response test signal [Fig. 2(d)] shows that the COIFM has a practical time resolution $\sim 1.5 \mathrm{~ms}$. The force resolution is less than $150 \mathrm{pN}$, which is higher force sensitivity by two orders of magnitude than the existing IFM with electrical detection method. ${ }^{9}$ The capability of this COIFM as a second generation of IFM has been demonstrated by revealing the hidden structures of the interfacial water on a silicon surface at the molecular scale. Figure 3(a) illustrates a typical force distance curve taken on a silicon surface (SPI Supplies) in air with feedback off as the tip approaches with the speed of $8 \mathrm{~nm} / \mathrm{s}$ at a distance of $50 \mathrm{~nm}$ away from the surface. In the force-displacement curve, the distance zero was defined as the intersection between the contact force line and the line where the interfacial force is zero. ${ }^{25} \mathrm{~A}$ long range repulsive force appears monotonously at the distances between 5 and $30 \mathrm{~nm}$ from the silicon surface, possibly resulting from the electrostatic dipole-dipole interaction observed by Kelvin probe measurement. ${ }^{26}$ The same experiment was repeated under a feedback-on condition. The voltage signal to the $\mathrm{ZnO}$ material $V_{\mathrm{ZnO}}$ and the error signal $V_{A-B}$ were recorded as a function of tip to sample distance, as shown in Figs. 3(b) and 3(c), respectively. One of the key features in Fig. 3(c) is that the $V_{A-B}$ voltage remains zero during approach, indicating that all forces on the cantilever remain balanced or the cantilever has "zero compliance" by relieving the strain built up in the $\mathrm{ZnO}$ stack through force feedback. However, the sensing cantilever starts to bend as soon as the tip touches the silicon surface indicating the break down of force feedback. The long range interaction is reproducibly obtained in to AlP license or copyright; see http://apl.aip.org/apl/copyright.jsp 
the force-distance curve with feedback on [Fig. 3(b)]. The background noise level $(0.1-0.2 \mathrm{nN})$ is smaller than the background noise $(1-2 \mathrm{nN})$ with feedback off by an order of magnitude.

Direct comparison between two force curves with feedback on and off in the distance range between 0 and $5 \mathrm{~nm}$ shows that fine periodic structures with several peaks and valleys appear from the surface in the force curve with feedback on, whereas, they are absent in the forcedisplacement curve with feedback off. Interestingly, the periodicity of peaks is $0.32 \pm 0.13 \mathrm{~nm}$ as marked with arrows in the detailed force-distance curve between 0 and $3 \mathrm{~nm}$ [inset of Fig. 3(b)], which is comparable with the diameter of a single water molecule. Similar periodic features have been observed by a few groups in recent years at interfaces between solid surfaces and liquid water using amplitude modulation methods, suggesting the possible ordering of water molecules near surfaces. ${ }^{27-30}$ This COIFM data on "interfacial" water demonstrates that the COIFM is capable of unveiling structural and mechanical information on interfacial water at the single molecular level, which has not been previously reported with the existing IFM. In contrast to the recent IFM studies of interfacial water reported with larger diameter tips $(1-10 \mu \mathrm{m}),{ }^{17,18}$ the sharp tip of the DMASP leads to probing the local structure of the interfacial water without averaging out the interfacial forces between the tip and the surface.

The $\mathrm{ZnO}$ feedback loop is capable of feeding back highfrequency signals (or small forces) due to the wide frequency response which is a hundred times larger than the $z$ bandwidth of the piezotube feedback loop of the ordinary AFM, allowing for more rapid, precise, and accurate force measurements than ordinary commercial AFM systems in the forcedistance curve. Instead of applying an opposing force on the force sensor through force feedback, as in the case of the existing IFM, the COIFM attains zero compliance by relieving the strain built on the cantilever. This feedback mechanism protects the tip from being damaged in conjunction with the flexible spring of DMASP, thus, allowing repeated use of the force sensor and improving reliability of the measurement.

The authors thank Joseph Holmes, J. J. Durrant, and Thanh Tran for all their help and support on this project. This research was supported by an award from the Research Corporation and NSF-Idaho EPSCoR Faculty Start-up Augmentation Fund.

${ }^{1}$ H. J. Butt, B. Cappella, and M. Kappl, Surf. Sci. Rep. 59, 1 (2005).

${ }^{2}$ I. Schumakovitch, W. Grange, T. Strunz, P. Bertoncini, H. J. Güntherodt, and M. Hegner, Biophys. J. 82, 517 (2002).

${ }^{3}$ J. Fritz, D. Anselmetti, J. Struct. Biol. 119, 165 (1997).

${ }^{4}$ B. Cappella and G. Dietler, Surf. Sci. Rep. 34, 1 (1999).

${ }^{5}$ J. A. Greenwood, Proc. R. Soc. London, Ser. A 453, 1277 (1997).

${ }^{6}$ A. Noy, D. V. Vezenov, and C. M. Lieber, Annu. Rev. Mater. Sci. 27, 381 (1997).

${ }^{7}$ J. N. Israelachvili and G. E. Adams, J. Chem. Soc., Faraday Trans. 1 74, 975 (1978)

${ }^{8}$ D. Sarid, Scanning Force Microscopy with Application to Electric, Magnetic and Atomic Forces (Oxford University Press, New York, 1991).

${ }^{9}$ S. A. Joyce and J. E. Houston, Rev. Sci. Instrum. 62, 710 (1991).

${ }^{10}$ J. E. Houston and T. A. Michalske, Nature (London) 356, 266 (1992).

${ }^{11}$ K. K. Chang, N. C. Shie, H. M. Tai, and T. L. Chen, J. Sci. Eng. 7, 91 (2004).

${ }^{12}$ A. R. Burns, J. E. Houston, R. W. Carpick, and T. A. Michalske, Langmuir 15, 2922 (1999).

${ }^{13}$ J. D. Kiely and J. E. Houston, Langmuir 22, 4513 (1999).

${ }^{14}$ H. I. Kim and J. E. Houston, J. Am. Chem. Soc. 122, 12045 (2000).

${ }^{15}$ B. C. Bunker, B. I. Kim, J. E. Houston, S. T. Picraux, R. Rosario, A. A. Garcia, M. Hayes, and D. Gust, Nano Lett. 3, 1723 (2003).

${ }^{16}$ D. L. Huber, R. P. Maginell, M. A. Samara, B. I. Kim, and B. C. Bunker, Science 301, 352 (2003).

${ }^{17}$ R. C. Major, J. E. Houston, M. J. McGrath, and X. Y. Zhu, Phys. Rev. Lett. 96, 177803 (2006).

${ }^{18}$ P. G. Matthew, J. E. Houston, and X. Y. Zhu, Langmuir 23, 5491 (2007).

${ }^{19}$ P. D. Ashby, L. Chen, and C. M. Lieber, J. Am. Chem. Soc. 122, 9467 (2000).

${ }^{20}$ G. Meyer and N. M. Amer, Appl. Phys. Lett. 53, 1045 (1988).

${ }^{21}$ A. M. Parker and J. L. Parker, Rev. Sci. Instrum. 63, 5626 (1992).

${ }^{22}$ S. P. Jarvis, H. Yamamoto, S. I. Yamamoto, H. Tokumoto, and J. B. Pethica, Nature (London) 384, 247 (1996).

${ }^{23}$ S. I. Yamamoto, H. Yamada, and H. Tokumoto, Rev. Sci. Instrum. 68, 4132 (1997)

${ }^{24}$ B. I. Kim, Rev. Sci. Instrum. 71, 5035 (2004).

${ }^{25}$ T. J. Senden, Curr. Opin. Colloid Interface Sci. 6, 95 (2001).

${ }^{26}$ A. Verdaguer, G. M. Sacha, H. Bluhm, and M. Salmeron, Chem. Rev. (Washington, D.C.) 106, 1478 (2006).

${ }^{27}$ M. Antognozzi, A. D. L. Humphris, and M. J. Miles, Appl. Phys. Lett. 78, 300 (2001)

${ }^{28}$ S. P. Jarvis, T. Uchihashi, T. Ishida, H. Tokumoto, and Y. Nakayama, J. Phys. Chem. B 104, 6091 (2000).

${ }^{29}$ T. Uchihashi, M. Higgins, Y. Nkayama, J. E. Sader, and S. P. Jarvis, Nanotechnology 16, S49 (2005).

${ }^{30}$ S. Jeffery, P. M. Hoffmann, J. B. Pethica, and C. Ramanujan, Phys. Rev. B 70, 054114 (2004). 